\title{
A continuous population of variable stars up to about 1.5 mag above the horizontal branch?
}

\author{
L. Baldacci ${ }^{1}$, G. Clementini ${ }^{2}$, E.V. Held ${ }^{3}$, M. Marconi ${ }^{4}$ L. Rizzi ${ }^{3}$ \\ 1 Bologna University, via Ranzani 1, I-40127 Bologna \\ 2 INAF - Bologna Observatory, via Ranzani 1 I-40127 Bologna \\ 3 INAF - Padova Observatory, vicolo dell'Osservatorio 5 I-35122 Padova \\ 4 INAF - Capodimonte Observatory, via Moiariello 16 I-80131 Napoli
}

\begin{abstract}
Increasing samples of pulsating variable stars populating the classical instability strip from the horizontal branch to a few magnitudes brighter are being found in several Local Group galaxies, irrespective of the galaxy morphological type. We will review the observational scenario focusing in particular on the Anomalous Cepheids and related objects.
\end{abstract}

\section{Introduction}

In recent years many Local Group (LG) galaxies have been surveyed looking for variable stars, however the observational scenario is rather inhomogeneous. In fact, while several of the dwarf Spheroidal (dSph) companions of the Milky Way had been studied in the early eighties, the advent of CCD detectors and wide field cameras, and the development of new powerful methods for the detection of the variables (e.g. the image subtraction techniques), have prompted for new surveys whose results, however, often are not yet published. On the other side, the study of the variable stars in the Irregular galaxies was impossible until a few years ago, except in the Magellanic Clouds. Thus recent and good quality photometric data exist only for a few of these galaxies. Moreover, the Magellanic Clouds benefited from the systematic surveys of the microlensing studies (e.g. MACHO: Alcock et al. 1996; OGLE: Udalski et al. 1997). Hence, samples for these two galaxies are much better studied and complete than for others.

A number of different types of pulsating variables lie in the portion of the HR diagram brighter than the RR Lyr stars. We will briefly describe the different types here.

Anomalous Cepheids (ACs) are metal poor $\left(Z \sim 10^{-4}\right)$ helium burning stars about 1 mag brighter than the RR Lyr stars, showing a range in period from $0.3 \mathrm{~d}$ to $2 \mathrm{~d}$. First observed in the Draco dSph by Baade \& Swope (1961), they occur in all the known dSphs (Pritzl et al. 2002, and references therein). On the contrary they are remarkably rare in Globular Clusters (GCs) where only one confirmed $\mathrm{AC}$ has been found in the low density globular cluster NGC 5466 (Zinn \& Dahn 1976). Zinn \& Searle (1976) named these variables Anomalous Cepheids because they fail to follow the period-luminosity $(\mathrm{P} / \mathrm{L})$ relation of Classical and Population II Cepheids (P2C). Because ACs do no show a different morphology of the light curves whether they pulsate in the fundamental or in the first overtone mode, the $\mathrm{P} / \mathrm{L}$ relations (Nemec et al. 1988, Nemec et al. 1994, Bono et al. 1997, Pritzl et al. 2002) are the only way to determine their pulsation mode, that, however, still remains uncertain for many of them. Theory and observations suggest that ACs are 2 or 3 times more massive than the RR Lyr 
stars, but we still lack precise estimates (Nemec et al. 1988 and references therein). Masses could allow us to disentangle between the two possible scenarios proposed for the origin of ACs: they are relatively young and massive stars ( $\sim 1$ Gyr, Norris \& Zinn $1975, M<2.5$ $\mathrm{M} \odot$ ) or they resulted from mass transfer in binary systems formed by old stars ( $\sim 10$ Gyrs, Renzini et al. 1977). In the latter case their masses could not exceed twice the turn-off mass of the system $(\sim 1.6 \mathrm{M} \odot$ for Spheroidal galaxies, see Wallerstein \& Cox 1984 for details). The question about the origin of the ACs in dSphs remains unsettled because none of these two hypotheses could be definitely ruled out (Da Costa 1988).

P2Cs are very common in Galactic GCs. They are very old, metal-poor, low mass $(\mathrm{M} \leq 0.6$ $\mathrm{M} \odot)$ stars that cross the instability strip while evolving from the blue tail of the HB towards the Asymptotic Giant Branch (see Wallerstein \& Cox, 1984). With the exception of Ursa Minor, dSphs generally do not have HBs extending to the blue enough to produce P2Cs, but they are found in the dSphs that host globular clusters, namely Fornax and Sagittarius.

Two further types of pulsating variables lie in the portion of the HR diagram brighter than the HB of the LG dwarf Irregular (dlrr) galaxies, they are the short period Cepheids (SPCs) and the low luminosity (LL) Cepheids. Smith et al. (1992) defined SPCs as a conspicuous population of variables in the Small Magellanic Cloud with periods between $0.6 \mathrm{~d}$ to $10 \mathrm{~d}$, that did not follow the $A C s P / L$ relations and fell instead on the extension to short periods of the Classical Cepheids' P/L relations. SPCs have been found in many dirrs since, they extend to longer periods and are brighter than the ACs. Both ACs and SPCs are helium burning stars, but the former have experienced the helium flash (namely have masses $M \leq 2.5 \mathrm{M}_{\odot}$ ), while the latter are blue loop stars that have ignited helium in non degenerate conditions $\left(M \geq 2.5 \mathrm{M}_{\odot}\right)$. SPCs should represent the low mass tail of the Classical Cepheids in low metallicity systems (Gallart et al. 1999, Dolphin et al. 2002).

LL Cepheids were observed for the first time in the dlrr galaxy NGC6822 by Clementini et al. (2003a). They have small amplitudes ( $0.1-0.4$ mag in V), are fainter and have shorter periods than the SPCs: they seem to merge in magnitude with the RR Lyr stars forming a continuum in the classical instability strip. Due to their small amplitudes, LL Cepheids are difficult to detect, thus they may have been missed in dlrr galaxies where crowding is severe. In the $\mathrm{P} / \mathrm{L}$ plane the LL Cepheids occupy the short-period low-luminosity region where ACs and Classic Cepheids $\mathrm{P} / \mathrm{L}$ relations merge (see fig. 2 ) and where the $4 \mathrm{ACs}$ detected in the LMC by Clementini et al. (2003b), and the SPCs recently found in Phoenix by Gallart et al. (2003) are also located. Because of their low luminosities LL Cepheids could be the counterpart of ACs in dlrrs, indeed models for ACs predict variables with such small amplitudes and low luminosities (Fiorentino et al. 2003).

\section{Dwarf Spheroidal Galaxies}

All dwarf Spheroidals studied so far are found to host ACs, although not in very large numbers (And VI: Pritzl et al. 2002; Leo II: Siegel \& Majewski 2000; Draco: Zinn \& Searle 1976, Kinemuchi et al. 2002; Ursa Minor: Nemec et al 1988; Carina: Saha et al. 1986, Dall'Ora et al. 2003; Sculptor: Swope 1968, Kaluzny et al. 1995; Sextans: Mateo et al. 1995a, Sagittarius: Mateo et al. 1995b, Layden \& Sarajedini 2000; AndlII: Da Costa et al. 2002; And II: Pritzl et al. 2003; Leo I: Hodge \& Wright 1978; Fornax: Light et al. 1986, Bersier \& Wood 2002, Clementini et al. 2003c, Mackey \& Gilmore 2003). Figure 1 shows the P/L relation of the $\mathrm{ACs}$ in the $\mathrm{V}$ band, drawn from the above literature data generally selecting only variables with accurate data. We have adopted the distance scale of Pritzl et al. (2002) which is consistent with a distance modulus of 18.5 for the LMC. The distance scale has a fundamental role both in the slope and zero point of the $A C s P / L$ relations (slanted solid lines in fig. 1), and in the pulsation mode determination. The horizontal lines mark the mean level of RR Lyr stars in the metal poorer (Ursa Minor) and the metal richer (Fornax) galaxies and show that there is no clear separation between the distributions of $A C s$ and $H B$ 


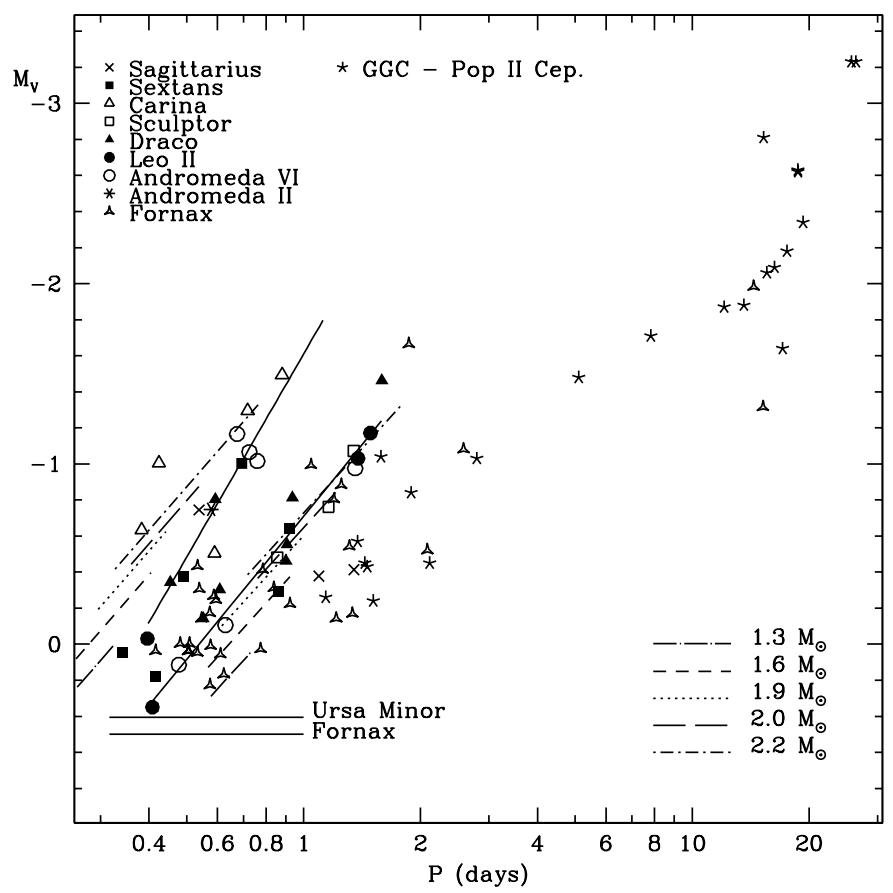

Figure 1: $\mathrm{P} / \mathrm{L}$ distribution of the $A C s$ in various $d S p h s$. The slanted solid lines are the $A C s P / L$ relations by Pritzl et al. (2002), the horizontal solid lines mark the RR Lyr average luminosity in Ursa Minor and Fornax. Theoretical boundaries of the ACs instability strips are from Marconi et al. (2004).

variables. Also shown in the figure are the boundaries of the theoretical instability strip of ACs models with different masses computed by Marconi et al. (2004). The Leol ACs are not displayed and are discussed more in detail in the next section. The Galactic GCs P2Cs collected by Nemec et al. (1994) are also shown in Figure 1. Fornax and Sagittarius are so far the only dSphs known to host GCs, and found to contain both ACs and P2Cs. Two of the variables in Sagittarius are in the cluster M54 (Layden \& Sarajedini 2000). They had been originally classified as a candidate $\mathrm{AC}$ and a candidate $\mathrm{P} 2 \mathrm{C}$, but clearly lie both in the P2Cs region. Fornax variables present a more complex scenario since many of the field variables classified as ACs by Bersier \& Wood (2000) lie instead on the P/L relation of the P2Cs. If confirmed when more accurate photometric data will become available, this would thus be the first identification of P2Cs in the field of a dSph.

\section{LeoI}

Leo I has a dominant young and intermediate-age stellar population. The galaxy has a bulk of candidate variables 2.4 mag brighter than the HB, and about 1 mag brighter than the ACs found in the other dSphs (Lee et al. 1993, Baldacci et al. 2003). Caputo et al. (1999) demonstrate that they are still consistent with ACs models, but Gallart et al. (1999) claim that they are bright enough to be SPCs. The published light curves for these variables (Hodge \& Wright 1978) are affected by a large scatter, thus the presence of variables more massive than the ACs in Leo I is a hypothesis that needs to be confirmed. 


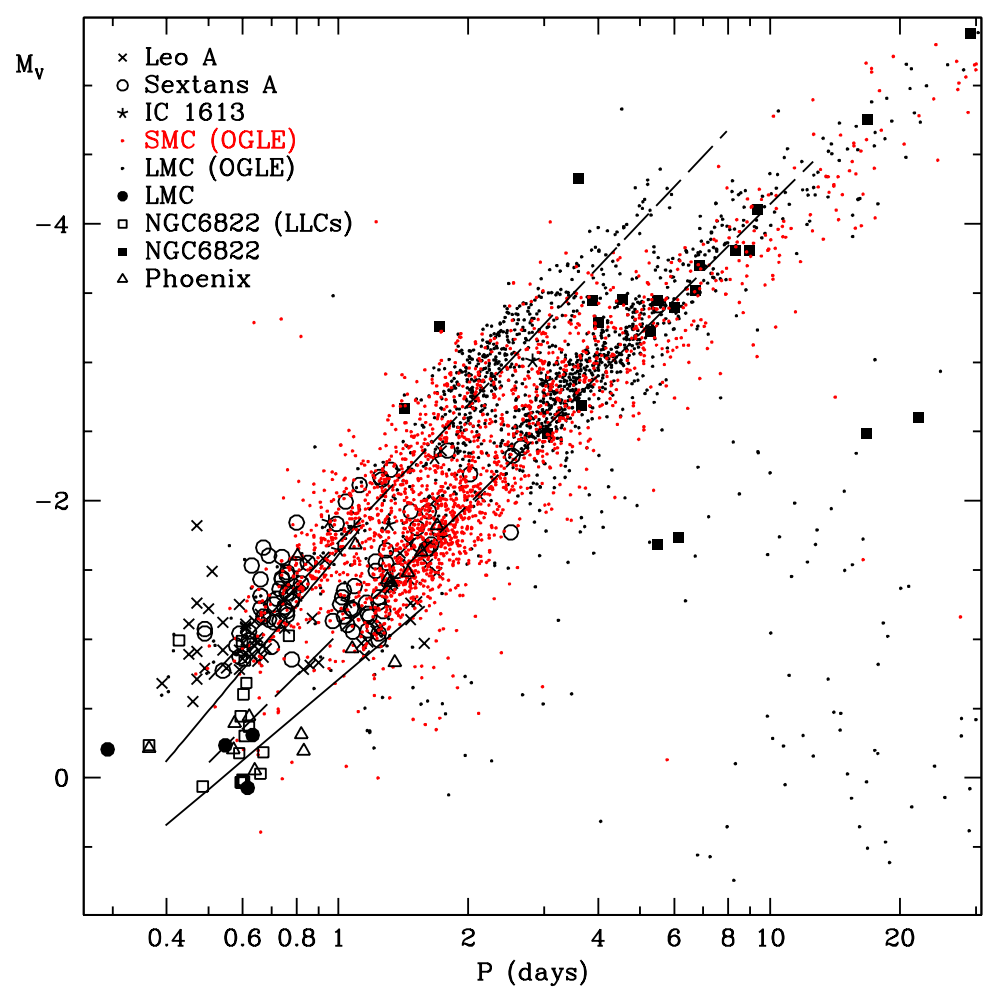

Figure 2: $\mathrm{P} / \mathrm{L}$ distribution of Cepheids with period shorter than $20 \mathrm{~d}$ found in LG Irregular galaxies.

\section{Dwarf Irregular Galaxies}

Figure 2 shows the $P / L$ distribution of Cepheids with period shorter than $20 \mathrm{~d}$ found in the LG Irregular galaxies (IC1613: Dolphin et al. 2001; Leo A: Dolphin et al. 2002; Sextans A: Dolphin et al. 2003; SMC: Udalski et al. 1999; LMC: Udalski et al. 1999, Clementini et al. 2003b; NGC6822: Antonello et al. 2002, Clementini et al. 2003a; Phoenix: Gallart et al. 2003). The dashed-dotted lines are the $P / L$ relations followed by the SPCs with periods shorter than 2 days in the SMC (see Dolphin et al. 2003 for details). We have extended them to larger periods (up to $\sim 10 \mathrm{~d}$ ) since they very well represent the general distributions shown in Figure 2. The solid lines are the $P / L$ relations for $A C s$ in $d S p h s$ by Pritzl et al. (2002). The $P / L$ relations of the first overtone ACs and Cepheids are similar to each other, the fundamental mode relations show instead some differences. The LL Cepheids in NGC6822 appear to be consistent both with the ACs and SPCs relations.

\section{Conclusion}

The classical instability strip appears to be continuously populated in the dlrrs with the LL Cepheids filling the gap between RR Lyr stars and SPCs. An instability strip uniformly 
populated as observed in NGC 6822 or in Phoenix probably reflects the continuous star formation process occurring in these galaxies. On the other side, ACs with luminosities close to the RR Lyr stars have been found in some dSphs but they are only a few. Thus they may represent the the tail at low masses of the ACs mass distribution, more than a continuity in the star formation process. The actual nature of the LL Cepheids still remains unclear since they cannot be distinguished from ACs and SPCs on the basis of the $\mathrm{P} / \mathrm{L}$ relations.

Acknowledgments. We are grateful to C. Gallart for providing us the data for the SPCs in Phoenix, and to D. Bersier for helpful discussions on the ACs and P2Cs in Fornax.

\section{References}

Alcock, C. et al. 1996, AJ 111, 1146

Antonello, E., Fugazza, D., Mantegazza, L., Stefanon, M., Covino, S. 2002, A\&A 386, 860

Baade, W., Swope, H.H. 1961, AJ 66, 300

Baldacci, L. et al. 2003, in "Stars in Galaxies", in press (astro-ph/0305506)

Bersier, D., Wood, P.R. 2002, AJ 123, 840

Bono, G., Caputo, F., Santolamazza, P., Cassisi, S., Piersimoni, A. 1997, AJ 113, 2209

Caputo, F., Cassisi, S., Castellani, M., Marconi, G., Santolamazza, P. 1999, AJ 117, 2199

Clementini, G., Held, E.V., Baldacci, L., Rizzi, L. 2003a, ApJ 588L, 85

Clementini, G. et al. 2003b, AJ 125, 1309

Clementini, G. et al. 2003c, in "Variable stars in the Local Group", in press (astro-ph/0310545)

Da Costa, G.S. 1988, IAU Symp. 126, 217

Da Costa, G.S., Armandroff, T.E., Caldwell, N. 2002, AJ 124, 332

Dall'Ora, M. et al. 2003, AJ 126, 197

Dolphin, A.E. et al. 2001, ApJ 550, 554

Dolphin, A.E. et al. 2002, AJ 123, 3154

Dolphin, A.E. et al. 2003, AJ 125, 1261

Fiorentino, G., Caputo, F., Marconi, M. 2003, in "Stars in Galaxies", in press

Gallart, C., Freedman, W.L., Aparicio, A., Bertelli, G., Chiosi, C., 1999, AJ 118, 2245

Gallart, C. et al. 2003, in "Variable stars in the Local Group", in press

Hodge, P.W., Wright, F.W. 1978, AJ 83, 228

Kaluzny, J. et al. 1995, A\&AS 112, 407

Kinemuchi, K. et al. 2002, ASP Conf. Ser. 259, 130

Layden, A.C., Sarajedini, A., 2000, AJ 119, 1760

Lee, M.G. et al. 1993, AJ 106, 1420

Light, R.M., Armandroff, T.E., Zinn, R. 1986, AJ 92, 43

Mackey, A.D., Gilmore, G.F., 2003, MNRAS, in press (astro-ph/0307275)

Marconi, M., Fiorentino, G., Caputo, F. 2004, A\&A, in press (astro-ph/0401332)

Mateo, M., Fischer, P., Krzeminski, W. 1995a, AJ 110, 2166

Mateo, M. et al. 1995b, AJ 110, 1141

Nemec, J.M., Wehlau, A., Mendes de Oliveira, C. 1988, AJ 96, 528

Nemec, J.M., Nemec, A.F.L., Lutz, T.E. 1994, AJ 108, 222

Norris, J., Zinn, R. 1975, ApJ 202, 335

Pritzl, B.J., Armandroff, T.E., Jacoby, G.H., Da Costa, G.S. 2002, AJ 124, 1464

Pritzl, B.J., Armandroff, T.E., Jacoby, G.H., Da Costa, G.S. 2003, AJ, in press (astro-ph 0310620)

Renzini, A., Mengel, J.G., Sweigart, A.V. 1977, A\&A 56, 369

Saha, A., Monet, D.G., Seitzer, P. 1986, AJ 92, 302

Siegel, M.H., Majewski, S.R 2000, AJ 120, 284

Swope, H.H. 1968, AJS 73, 204

Smith, H.A., Silbermann, N.A., Baird, S.R., Graham, J.A. 1992, AJ 104, 1430

Udalski, A., Kubiak, M., Szymanski, M. 1997, AcA 47, 319

Udalski, A. et al. 1999, AcA 49, 201

Wallerstein, G., Cox, A.N. 1984, PASP 96, 677

Zinn, R., Dahn, C.C. 1976, AJ 81,527

Zinn, R., Searle, L. 1976, ApJ 209, 734 